\title{
Evaluation of portable microscopic devices for the diagnosis of Schistosoma and soil-transmitted helminth infection
}

\author{
ISAAC I. BOGOCH ${ }^{1} *$, JEAN T. COULIBALY ${ }^{2,3}$, JASON R. ANDREWS ${ }^{4}$, BENJAMIN $^{5}$ \\ SPEICH $^{5,6}$, JENNIFER KEISER ${ }^{5,6}$, J. RUSSELL STOTHARD ${ }^{7}$, ELIÉZER K. N'GORAN ${ }^{2,3}$ \\ and JÜRG UTZINGER ${ }^{6,8}$ \\ ${ }^{1}$ Divisions of Internal Medicine and Infectious Diseases, Toronto General Hospital, 14EN-209, 200 Elizabeth Street, \\ Toronto, ON, M5G 2C4, Canada \\ ${ }^{2}$ Unité de Formation et de Recherche Biosciences, Université Félix Houphouët-Boigny, 22 BP 770, Abidjan 22, Côte d'Ivoire \\ ${ }^{3}$ Centre Suisse de Recherches Scientifiques en Côte d'Ivoire, 01 BP 1303, Abidjan 01, Côte d'Ivoire \\ ${ }^{4}$ Division of Infectious Diseases and Geographic Medicine, Stanford University School of Medicine, Stanford, CA, \\ 94305-5107, USA \\ ${ }^{5}$ Department of Medical Parasitology and Infection Biology, Swiss Tropical and Public Health Institute, P.O. Box, \\ CH-4002 Basel, Switzerland \\ ${ }^{6}$ University of Basel, P.O. Box, CH-4003 Basel, Switzerland \\ ${ }^{7}$ Parasitology Department, Liverpool School of Tropical Medicine, Liverpool L3 5QA, UK \\ ${ }^{8}$ Department of Epidemiology and Public Health, Swiss Tropical and Public Health Institute, P.O. Box, CH-4002 Basel, \\ Switzerland
}

(Received 1 February 2014; revised 27 February 2014; accepted 28 February 2014; first published online 24 April 2014)

\section{SUMMARY}

The diagnosis of parasitic worm (helminth) infections requires specialized laboratory settings, but most affected individuals reside in locations without access to such facilities. We tested two portable microscopic devices for the diagnosis of helminth infections in a cross-sectional survey in rural Côte d'Ivoire. We examined 164 stool samples under a light microscope and then re-examined with a commercial portable light microscope and an experimental mobile phone microscope for the diagnosis of Schistosoma mansoni and soil-transmitted helminths. Additionally, 180 filtered urine samples were examined by standard microscopy and compared with the portable light microscope for detection of Schistosoma haematobium eggs. Conventional microscopy was considered the diagnostic reference standard. For S. mansoni, S. haematobium and Trichuris trichiura, the portable light microscope showed sensitivities of $84 \cdot 8 \%, 78 \cdot 6 \%$ and $81 \cdot 5 \%$, respectively, and specificities of $85.7 \%, 91 \cdot 0 \%$ and $93.0 \%$, respectively. For S. mansoni and T. trichiura, we found sensitivities for the mobile phone microscope of $68 \cdot 2 \%$ and $30 \cdot 8 \%$, respectively, and specificities of $64 \cdot 3 \%$ and $71 \cdot 0 \%$, respectively. We conclude that the portable light microscope has sufficient diagnostic yield for Schistosoma and T. trichiura infections, while the mobile phone microscope has only modest sensitivity in its current experimental set-up. Development of portable diagnostic technologies that can be used at point-of-sample collection will enhance diagnostic coverage in clinical and epidemiological settings.

Key words: Diagnosis, microscopy, mobile phone, schistosomiasis, soil-transmitted helminths.

\section{INTRODUCTION}

Schistosomiasis (caused by chronic infection with blood-dwelling flukes of the genus Schistosoma) and soil-transmitted helminthiasis (caused by infections with nematode worms Ascaris lumbricoides, Trichuris trichiura and hookworm) remain important public health issues, particularly in the tropics and subtropics (Hotez et al. 2008; Murray et al. 2012). More than $90 \%$ of the estimated 207 million individuals infected with Schistosoma reside in Africa (Steinmann et al. 2006). Chronic infection with Schistosoma mansoni leads to morbidity and mortality from portal hypertension, while chronic Schistosoma

\footnotetext{
* Corresponding author. Divisions of Internal Medicine and Infectious Diseases, Toronto General Hospital, 14EN209, 200 Elizabeth Street, Toronto, ON, M5G 2C4, Canada.E-mail: isaac.bogoch@uhn.ca
}

haematobium infection is associated with genitourinary pathology which may result in hydronephrosis, renal failure and squamous cell bladder cancer (Ross et al. 2002; Gryseels et al. 2006). Over a billion people are infected with soil-transmitted helminths, with school-aged children and marginalized communities in resource-constrained settings disproportionately affected. Chronic soil-transmitted helminth infection may result in anaemia, malnutrition and impaired physical and cognitive development (Bethony et al. 2006). In the developing world, infection with multiple species is the norm rather than the exception (Keiser et al. 2002; Steinmann et al. 2010).

The diagnosis of S. mansoni and soil-transmitted helminth infections traditionally and functionally relies on stool microscopy, with the Kato-Katz technique being widely used, although the method has important shortcomings (Katz et al. 1972; 
Kongs et al. 2001; Booth et al. 2003; Speich et al. 2010). Indeed, it is a relatively inexpensive and simple method of stool preparation, however multiple Kato-Katz thick smears are required to increase diagnostic sensitivity, particularly for low-intensity infections (de Vlas and Gryseels, 1992; Knopp et al. 2008). Schistosoma haematobium is traditionally diagnosed by urine microscopy, using filtration or sedimentation techniques (Knopp et al. 2013). Indirect methods such as urine reagent strips assessing for microhaematuria have been widely used in endemic settings, albeit with variable estimates of sensitivity and specificity (Mott et al. 1983; Robinson et al. 2009; Kosinski et al. 2011; Bogoch et al. 2012).

Most individuals infected with Schistosoma and soil-transmitted helminths live in rural, resourceconstrained settings where facilities to perform the aforementioned diagnostic tests are often nonexistent. Additional barriers to care include the transport of patients or biological samples to functional diagnostic centres and a constant electricity supply for light microscopy. Hence, alternative diagnostic approaches are necessary to better care for affected individuals living in endemic settings. Portable light microscopy for S. mansoni diagnosis has been used with some success in a pilot study carried out in Uganda (Stothard et al. 2005). In addition, mobile phone microscopy was recently shown to have modest diagnostic yield for the diagnosis of soil-transmitted helminth infections in school-aged children in a proof-of-concept study on Pemba Island, Tanzania (Bogoch et al. 2013). These diagnostic innovations have the benefit of being portable, inexpensive, easy to use, point-of-care tests that do not require a constant electricity supply.

The purpose of this study was to assess the utility of a novel commercial, portable light microscope and a simple mobile phone microscope for the diagnosis of $S$. mansoni, S. haematobium and soil-transmitted helminths. The diagnostic performance is compared with microscopy using standard Kato-Katz and urine filtration methods considered as referencestandard.

\section{MATERIALS AND METHODS}

\section{Ethics statement}

This study was integrated into a cross-sectional survey in Côte d'Ivoire, and was approved by the ethics committee of the Ministry of Health and Public Hygiene in Côte d'Ivoire (reference no. 7706/ MSLS/CNER-dkn). Written informed consent was obtained from parents or legal guardians on behalf of children, and children assented orally. At the end of the study, anthelmintic treatment was offered to all participants regardless of their infection status (i.e. praziquantel, $40 \mathrm{mg} \mathrm{kg}^{-1}$ of body weight against schistosomiasis; albendazole, $400 \mathrm{mg}$ against soiltransmitted helminthiasis).

\section{Population census}

The study was conducted among school-aged children residing in Azaguié Makouguié, a village of the district of Azaguié, south Côte d'Ivoire, where S. mansoni, S. haematobium and soil-transmitted helminths are co-endemic (Coulibaly et al. 2012, 2013). A census was carried out in May 2013. All households were visited and demographic characteristics were collected from school-aged children, including name, age and sex.

\section{Study design and procedures}

Children were invited for multiple stool and urine sample collection over a 3-day period. Urine sample collection was done between 10:00 and 12:00 h, and fresh stool samples were collected from the early morning on the date of analysis in the laboratory from the cohort. Samples were transferred to a nearby laboratory in the town of Azaguié and processed the same day using standard protocols.

In brief, stool samples were subjected to the KatoKatz technique (Katz et al. 1972), preparing five $41.7 \mathrm{mg}$ thick smears on microscope slides which were examined under a CX21LEDFS1 Olympus microscope (Olympus; Volketswil, Switzerland) by experienced laboratory technicians with $20 \times$ and $40 \times$ objective lenses. The number of $S$. mansoni, A. lumbricoides, hookworm and T. trichiura eggs were counted and recorded for each species separately. Urine samples were processed by a filtration technique for evaluation and quantification of S. haematobium eggs (Peters et al. 1976). Briefly, urine samples were shaken prior to extracting and pressing $10 \mathrm{~mL}$ through a meshed filter $(20 \mu \mathrm{M}$ pores $)$ measuring $13 \mathrm{~mm}$ in diameter (Sefar AG; Heiden, Switzerland). One drop of Lugol's iodine solution was placed over the filter prior to microscopic examination. For quality control, $10 \%$ of all Kato-Katz thick smears and urine filtrations were re-examined by a senior microscopist.

For the purpose of the current embedded study, we selected the first out of five Kato-Katz thick smears obtained from the third-day stool sample collection $(n=164)$ for further microscopic evaluation. Given the low prevalence of $S$. haematobium in the study village (approximately 5\%), we selected all positive samples from each day of collection, resulting in a total of 14 positive slides. Then, randomly selected negative slides were added to total up to 60 slides from each day of collection, such that there were 180 slides overall. Our evaluation with experimental microscopic techniques occurred 10-12 days after initial sample collection and slide preparation.

\section{Microscopic techniques}

A mobile phone microscope was constructed and utilized as described previously (Smith et al. 2011; 
Bogoch et al. 2013). Briefly, a $3 \mathrm{~mm}$ glass ball lens (Edmund Optics; Barrington, NJ, USA) was mounted over the camera lens of an iPhone $4 \mathrm{~S}$ (Apple; Cupertino, CA, USA), with $1 / 16^{\prime \prime}$ thick foam tape (AM Rubber; Canada). Slides were placed up against the mobile phone, such that the tape provided approximately a $1 \mathrm{~mm}$ buffer zone between the slide and the ball lens. Slides were illuminated from below with a hand-held incandescent flashlight powered by one AA battery. The iPhone was manually manoeuvred over the entire slide to systematically search for helminth eggs using the camera function and with the aid of auto focus and digital zoom functions.

Kato-Katz thick smears and filtered urine slides were also evaluated with the Newton Nm1-600 XY Portable Field Microscope (Newton Microscopes; Cambridge, UK). This is a commercially available, hand-held light microscope weighing $480 \mathrm{~g}$ with $10 \times, 40 \times$ and $60 \times$ objective lenses (Newton Microscopes). The objective lenses are modular such that the device can accommodate lenses with greater magnification as well. The same experienced microscopist evaluated all slides for the presence or absence of helminth eggs with the Newton portable microscope and mobile phone microscope. Infection intensity was not quantified. The microscopist was blinded to prior results from conventional microscopy.

\section{Statistical analysis}

Data were entered into a Microsoft Excel file (Microsoft; Redmond, WA, USA) and analysed with Stata version 10.1 (StataCorp.; College Station, TX, USA). The sensitivity, specificity, positive predictive value (PPV) and negative predictive value (NPV) were calculated for each helminth species using the iPhone microscope and the Newton microscope and compared with conventional light microscopy, with the latter serving as diagnostic reference standard. We then evaluated the diagnostic characteristics of the experimental microscopes for low, moderate and heavy infection intensity as per the cut-offs described by the World Health Organization (WHO, 2002), with moderate- and high-intensity infections grouped together. Two-sample tests of proportion were conducted to first compare the sensitivities and specificities of the experimental diagnostic techniques with reference-standard light microscopy and then compare the sensitivities between moderate/heavy infection intensities from light infections.

\section{RESULTS}

\section{Stool examination}

Data from the evaluation of 164 Kato-Katz thick smears with mobile phone microscopy and portable field microscopy $v s$ standard microscopy are presented in Table 1. The observed prevalence of S. mansoni and T. trichiura in this cohort based on a single Kato-Katz thick smear examined under traditional light microscopy was $91 \cdot 5 \%$ and $39 \cdot 4 \%$, respectively. No other helminths were visualized during the immediate evaluation with conventional light microscopy and subsequent evaluation with portable microscopic devices. Images can be viewed in Fig. 1. S. mansoni, S. haematobium and T. trichiura eggs can be easily visualized with conventional microscopy and the Newton portable microscope, but are more challenging to see with the mobile phone microscope.

For S. mansoni and T. trichiura, the Newton portable microscope showed sensitivities of $84.8 \%$ and $81 \cdot 5 \%$, respectively, and specificities of $85.7 \%$ and $93.0 \%$, respectively (Table 1 ). For S. mansoni and T. trichiura the mobile phone microscope showed sensitivities of $68 \cdot 2 \%$ and $30 \cdot 8 \%$, respectively, and specificities of $64 \cdot 3 \%$ and $71 \cdot 0 \%$, respectively. Compared with the iPhone microscope, the Newton portable microscope had greater sensitivity $(P=0.001)$ but not specificity $(P=0.383)$ for the diagnosis of $S$. mansoni. For T. trichiura infection, the Newton portable microscope had both greater sensitivity and specificity (both $P$ values $<0 \cdot 001$ ) compared with the iPhone.

For the evaluation of infection intensities, the iPhone demonstrated greater sensitivity for moderate/heavy infections compared with light infections of $S$. mansoni $(P<0 \cdot 001)$ and T. trichiura $(P=0 \cdot 019)$. The Newton portable microscope's sensitivity fared better for moderate/heavy infection intensity compared with light infections with $S$. mansoni $(P<0.001)$ and similarly with all infection intensities for $T$. trichiura infections $(P=0 \cdot 323)$.

\section{Urine examination}

Overall, $8 \cdot 3 \%$ of urine slides included in this comparison were positive for $S$. haematobium by light microscopy. The iPhone mobile microscope was used only in the evaluation of Kato-Katz thick smears, and was abandoned for urine filtration slides as it was unable to reliably detect eggs of $S$. haematobium during the pilot study, despite multiple attempts (see Discussion for details). Evaluation of samples with the Newton portable field microscope resulted in sensitivity and specificity of $78.6 \%$ and $91.0 \%$, respectively, for $S$. haematobium diagnosis (Table 1). This device did not confirm any statistically significant differences in diagnostic sensitivity for light or moderate/heavy infection intensity $(P=0 \cdot 154)$.

\section{DISCUSSION}

Scale-up of diagnostic coverage in rural areas of Africa will be necessary for a more accurate 
Table 1. Operating characteristics of the mobile phone microscope and Newton portable hand-held microscope compared with conventional light microscopy for the diagnosis of $S$. mansoni, T. trichiura and S. haematobium ( $n=164$ Kato-Katz thick smears; $n=180$ slides with filtered urine)

\begin{tabular}{|c|c|c|c|c|c|c|c|c|c|}
\hline \multirow[b]{2}{*}{ Organism } & \multirow[b]{2}{*}{$\begin{array}{l}\text { Conventional } \\
\text { microscope } n(\%)\end{array}$} & \multicolumn{4}{|c|}{ Newton Nm1-600 XY portable field microscope } & \multicolumn{4}{|c|}{ Mobile phone microscope } \\
\hline & & $\begin{array}{l}\text { Sensitivity } \\
(95 \% \mathrm{CI})\end{array}$ & $\begin{array}{l}\text { Specificity } \\
(95 \% \text { CI })\end{array}$ & $\begin{array}{l}\text { PPV } \\
(95 \% \mathrm{CI})\end{array}$ & $\begin{array}{l}\text { NPV } \\
(95 \% \text { CI })\end{array}$ & $\begin{array}{l}\text { Sensitivity } \\
(95 \% \mathrm{CI})\end{array}$ & $\begin{array}{l}\text { Specificity } \\
(95 \% \text { CI })\end{array}$ & $\begin{array}{l}\text { PPV } \\
(95 \% \mathrm{CI})\end{array}$ & $\begin{array}{l}\text { NPV } \\
(95 \% \text { CI })\end{array}$ \\
\hline Any helminth on Kato-Katz & $154(93 \cdot 3)$ & $88 \cdot 3(82 \cdot 2-92 \cdot 9)$ & $81 \cdot 8(48 \cdot 2-97 \cdot 7)$ & $98 \cdot 6(94 \cdot 9-99 \cdot 8)$ & $33 \cdot 3(16 \cdot 5-54 \cdot 0)$ & $74 \cdot 7(67 \cdot 0-81 \cdot 3)$ & $36 \cdot 4(10 \cdot 9-69 \cdot 2)$ & $94 \cdot 3(88 \cdot 5-97 \cdot 7)$ & $9 \cdot 3(2 \cdot 6-22 \cdot 1)$ \\
\hline S. mansoni (all) & $151(91 \cdot 5)$ & $84 \cdot 8(78 \cdot 0-90 \cdot 1)$ & $85 \cdot 7(57 \cdot 2-98 \cdot 2)$ & $98 \cdot 5(94 \cdot 6-99 \cdot 8)$ & $34 \cdot 3(19 \cdot 1-52 \cdot 2)$ & $68 \cdot 2(60 \cdot 1-75 \cdot 5)$ & $64 \cdot 3(35 \cdot 1-87 \cdot 2)$ & $95 \cdot 4(89 \cdot 5-98 \cdot 5)$ & $15 \cdot 8(7 \cdot 5-27 \cdot 9)$ \\
\hline S. mansoni (light $)^{\mathrm{a}}$ & $31(18 \cdot 8)$ & $45 \cdot 2(27 \cdot 8-63 \cdot 7)$ & - & - & - & $29 \cdot 0(14 \cdot 9-48 \cdot 2)$ & - & - & - \\
\hline $\begin{array}{l}\text { S. mansoni (moderate/ } \\
\text { heavy) }\end{array}$ & $120(72 \cdot 7)$ & $95 \cdot 0(89 \cdot 0-98 \cdot 0)$ & - & - & - & $78 \cdot 3(70 \cdot 0-85 \cdot 1)$ & - & - & - \\
\hline T. trichiura (all) & $65(39 \cdot 4)$ & $81 \cdot 5(70 \cdot 0-90 \cdot 1)$ & $93 \cdot 0(86 \cdot 1-97 \cdot 1)$ & $88 \cdot 3(77 \cdot 4-95 \cdot 2)$ & $88 \cdot 6(80 \cdot 9-94 \cdot 0)$ & $30 \cdot 8(19 \cdot 9-43 \cdot 4)$ & $71 \cdot 0(61 \cdot 1-79 \cdot 6)$ & $40 \cdot 8(27 \cdot 0-55 \cdot 8)$ & $61 \cdot 2(51 \cdot 7-70 \cdot 1)$ \\
\hline T. trichiura $(\text { light })^{\mathrm{c}}$ & $57(34 \cdot 5)$ & $80 \cdot 7(67 \cdot 7-89 \cdot 5)$ & - & - & - & $26 \cdot 3(15 \cdot 9-39 \cdot 9)$ & - & - & - \\
\hline $\begin{array}{l}\text { T. trichiura (moderate/ } \\
\text { heavy) }\end{array}$ & $8(4 \cdot 8)$ & $87 \cdot 5(46 \cdot 7-99 \cdot 3)$ & - & - & - & $62 \cdot 5(25 \cdot 9-89 \cdot 8)$ & - & - & - \\
\hline S. haematobium (all) & $14(7 \cdot 8)$ & $78 \cdot 6(49 \cdot 2-95 \cdot 3)$ & $91 \cdot 0(85 \cdot 5-94 \cdot 9)$ & $42 \cdot 3(23 \cdot 4-63 \cdot 1)$ & $98 \cdot 1(94 \cdot 4-99 \cdot 6)$ & - & - & - & - \\
\hline S. haematobium (light) ${ }^{\mathrm{e}}$ & $11(6 \cdot 1)$ & $72 \cdot 7(39 \cdot 3-92 \cdot 7)$ & - & - & - & - & - & - & - \\
\hline S. haematobium (heavy) ${ }^{\mathrm{f}}$ & $3(1 \cdot 7)$ & $100(-)$ & - & - & - & - & - & - & - \\
\hline
\end{tabular}

$\mathrm{CI}=$ confidence interval $\mathrm{PPV}=$ positive predictive value; $\mathrm{NPV}=$ negative predictive value.

a Eggs per $1 \mathrm{~g}$ of stool (EPG): 1-99.

b $E P G \geqslant 100$.

c EPG 1-999.

${ }^{\mathrm{d}} \mathrm{EPG} \geqslant 1000$

Eggs per $10 \mathrm{~mL}$ urine: $1-49$

Eggs per $10 \mathrm{~mL}$ urine: $\geqslant 50$ 


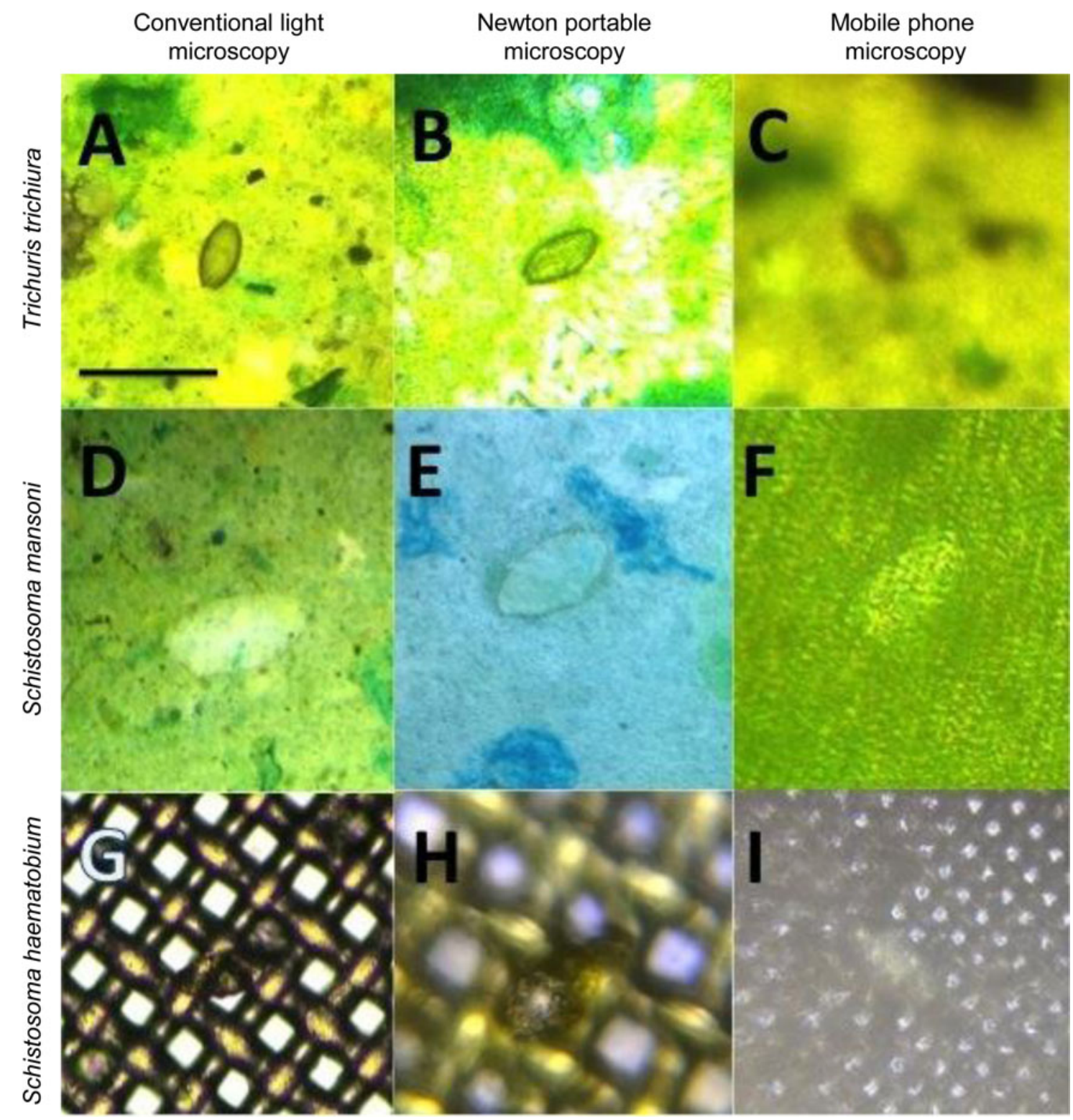

Fig. 1. Trichuris trichiura imaged by (A) conventional light microscopy, (B) Newton portable microscopy and (C) mobile phone microscopy; Schistosoma mansoni, imaged by (D) conventional light microscopy, (E) Newton portable microscopy and (F) mobile phone microscopy. Schistosoma haematobium imaged by (G) conventional light microscopy, (H) Newton portable microscopy and (I) mobile phone microscopy. All images were captured using the camera function of the iPhone 4S (Apple; Cupertino, CA, USA).

assessment of the impact of preventive chemotherapy campaigns against common parasitic worm infections and may help with direct patient care in under-resourced clinics. We demonstrate the utility of portable, hand-held microscopic techniques for the diagnosis of S. mansoni, S. haematobium and soiltransmitted helminths in a cohort of school-aged children from Côte d'Ivoire.

These diagnostic tools were evaluated in a village that has previously benefited from country-wide helminth control efforts targeting school-aged children as per WHO guidelines. Despite these efforts, there was still a very high prevalence of $S$. mansoni, with lower prevalences of $T$. trichiura and $S$. haematobium, while neither $A$. lumbricoides nor hookworm infection were found during the initial immediate evaluation with conventional light microscopy or subsequent portable microscopic devices. The prevalence of these infections would likely be higher if multiple slides were examined (Utzinger et al. 2001; Booth et al. 2003; Knopp et al. 2008) (e.g. five slides were prepared from each stool sample in this study), however the purpose of this embedded study was to compare microscopic techniques on the same slide set. The Newton portable light microscope performed with sufficient diagnostic sensitivity and specificity for S. mansoni, $S$. haematobium and $T$. trichiura such that it could be considered a valuable diagnostic device in future clinical and epidemiological studies. The mobile 
phone microscope performed modestly well with moderate- to heavy-intensity infections of $S$. mansoni and $T$. trichiura, but did not have acceptable diagnostic accuracy with light infections. The specificity for both S. mansoni and T. trichiura was unacceptably low in the current configuration.

We found the Newton portable microscope to be an effective diagnostic tool. It is compact, weighs less than $500 \mathrm{~g}$, and has an easy-to-use design for evaluating slides under objective lenses of differing magnification. The device requires three AAA batteries that can provide over $300 \mathrm{~h}$ of use. Images were easily captured by using a mobile phone with a camera function, and placing the lens of the camera directly up to the optical tube (Fig. 1B, E and H), however a specific mobile phone attachment designated for this purpose can be used as well. Also helpful was the ability to move slides to examine multiple fields, a feature not seen on previous portable microscopes used for field diagnoses of schistosomiasis (Stothard et al. 2005). This allows for the complete evaluation of a Kato-Katz thick smear or a urine filtration sample, and should decrease the number of false-negative results as eggs on the periphery of the slide will likely be visualized. Negative aspects of this device include its cost. The Newton microscope costs $£, 580$ ( US\$950) in highincome countries and $£ 250$ ( US $\$ 410$ ) in low- and middle-income countries at the time of writing. Fortunately, the prices of these microscopes and smartphones with a suitable camera function have declined and will do so in the future to make these technologies more affordable, perhaps helping the adoption and coverage of this technology in helminth-endemic settings. In contrast, quality light microscopes in laboratory settings as used in the current study are considerably more expensive ( US $\$ 1750$ for the CX21SEDFS1 model of Olympus microscope employed here). In addition, the diagnostic sensitivity was low for light-intensity infections, which may pose problems for evaluating the affects of preventative chemotherapy campaigns. Another disadvantage is that slides must be placed with the specimen side facing downward. While we had no issues with downward facing slides in this study, problems could theoretically arise related to sample loss and associated hygienic complications, such as biological material contaminating the microscope, or even worse, contaminating subsequent slides placed on the microscope. Lastly, digitization of the images is an important component of portable diagnostic devices (Tuijn et al. 2011) and the Newton portable microscope requires an additional piece of equipment such as a camera or a mobile phone with camera function to capture images, adding to the overall costs. We did not quantify egg counts on slides, and future studies evaluating this device should do so as this information is of critical importance from clinical, epidemiological and public health points of view. Additional studies should also evaluate the diagnostic capacity of the device when used by laboratory technicians and other medical personnel (e.g. nurses, primary care physicians) in routine practice, rather than expert microscopists.

In a previous study, mobile phone microscopy demonstrated an overall diagnostic sensitivity for T. trichiura of $54 \cdot 4 \%$, and sensitivities of $76 \cdot 5 \%$ and $43.9 \%$ for moderate-to-heavy infections and light infections, respectively (Bogoch et al. 2013). The sensitivities for $T$. trichiura are comparable in this study, albeit slightly lower, which may be related to a greater prevalence of lighter-intensity infections in the current study. As per previous studies with mobile phone microscopy, the diagnostic utility was greater with moderate-to-heavy infection intensities regardless of the helminth species. In addition, the larger eggs of $S$. mansoni may be easier to identify than $T$. trichiura, perhaps accounting for the relative greater sensitivity for this infection. The Newton portable microscope demonstrated no significant differences between light and moderate/heavy infections for $T$. trichiura or $S$. haematobium as the sensitivities were already quite high for lightintensity infections and because sample size was relatively small.

We had considerable difficulty obtaining clear images of $S$. haematobium with the mobile phone microscope during the pilot phase of this project such that data were not collected due to our inability to reliably capture images (e.g. Fig. 1I). Images appear to be 'washed out' perhaps from light refracting off the fine-meshed filter paper used to process urine. We used meshed filter paper with $20 \mu \mathrm{m}$ pores (Sefar AG; Heiden, Switzerland), which are less expensive than the more widely used Millipore filters (Millipore Intertech; Bedford, MA) (Gyorkos et al. 2001). We attempted different strategies of illuminating the specimen (e.g. varying intensities of light from different angles and using sunlight) but could not reliably capture detailed images. These issues did not arise with the Newton portable microscope. We also attempted to use a commercially available mobile phone microscope attachment (the American 3B Scientific iPhone Microscope; American 3B Scientific, Tucker, GA, USA) on both stool and urine samples during the pilot period, but found the device to be too cumbersome to reliably provide images of sufficient diagnostic quality at an appropriate magnification (3B Scientific).

The diagnostic yield of our mobile phone microscope in its current formulation is not sufficient for routine use in clinical or epidemiological settings, but perhaps can serve as a platform for future innovative devices. Several newer mobile phone technologies have shown promise for point-of-care diagnostic testing, including lens-free devices that provide a wide field of view for image analysis (Lee et al. 2011; Greenbaum et al. 2012; Isikman et al. 2012; 
Linder et al. 2013). Such devices may not be far from routine use in clinical or epidemiological settings as they are relatively inexpensive and have decent operating characteristics, but will require rigorous validation outside of laboratory settings prior to scale-up. Similarly, commercially available mobile phone microscope attachments may also be useful for global health applications, although in our experience several of the devices currently on the market either do not have the appropriate magnification or are too cumbersome to use in routine practice. Digitization of samples, and tagging these with geographical coordinates may identify regions with a higher prevalence of infection, and may facilitate the rapid and real-time reporting of cross-sectional surveys in endemic regions. It may also play a role in medical education as images may be shared readily with experts or an automated device such that real-time feedback is provided to the microscopist (Tuijn et al. 2011). Other low-cost diagnostic devices may be applicable for global health applications, such as the urine circulating cathodic antigen test for S. mansoni, a point-of-care test with excellent sensitivity (Coulibaly et al. 2011; Colley et al. 2013).

Portable diagnostic devices hold promise for enabling care in remote and resource-constrained settings. The Newton portable microscope demonstrated sufficient diagnostic yield of $S$. mansoni, $S$. haematobium and $T$. trichiura compared with standard light microscopy and should be studied further in different clinical and epidemiological settings. Our mobile phone microscope performed only modestly well at moderate-to-high infection intensities for S. mansoni and T. trichiura, however newer, low-cost technologies are in the pipeline for global health applications.

\section{ACKNOWLEDGEMENTS}

We thank the village authorities of Azaguié Makouguié and the participating children for providing stool and urine samples for detailed laboratory work-up. J. T. Coulibaly is grateful to the Swiss Tropical and Public Health Institute for a postdoctoral fellowship.

\section{FINANCIAL SUPPORT}

The research of B. Speich is financially supported by the Medicor Foundation, and that of J. Keiser, E. K. N'Goran and J. Utzinger by the Swiss National Science Foundation.

\section{REFERENCES}

Bethony, J., Brooker, S., Albonico, M., Geiger, S. M., Loukas, A., Diemert, D. and Hotez, P. J. (2006). Soil-transmitted helminth infections: ascariasis, trichuriasis, and hookworm. Lancet 367, 1521-1532.

Bogoch, I. I., Andrews, J. R., Dadzie Ephraim, R. K. and Utzinger, J. (2012). Simple questionnaire and urine reagent strips compared to microscopy for the diagnosis of Schistosoma haematobium in a community in northern Ghana. Tropical Medicine and International Health. doi: 10.1111/j.1365-3156.2012.03054.x.
Bogoch, I. I., Andrews, J. R., Speich, B., Utzinger, J., Ame, S. M., Ali, S. M. and Keiser, J. (2013). Mobile phone microscopy for the diagnosis of soil-transmitted helminth infections: a proof-of-concept study. American Fournal of Tropical Medicine and Hygiene 88, 626-629.

Booth, M., Vounatsou, P., N'Goran, E. K., Tanner, M. and Utzinger, J. (2003). The influence of sampling effort and the performance of the Kato-Katz technique in diagnosing Schistosoma mansoni and hookworm co-infections in rural Côte d'Ivoire. Parasitology 127, 525-531. Colley, D. G., Binder, S., Campbell, C., King, C. H., Tchuem Tchuenté, L. A., N'Goran, E. K., Erko, B., Karanja, D. M., Kabatereine, N. B., van Lieshout, L. and Rathbun, S. (2013). A fivecountry evaluation of a point-of-care circulating cathodic antigen urine assay for the prevalence of Schistosoma mansoni. American Fournal of Tropical Medicine and Hygiene 88, 426-432.

Coulibaly, J. T., Knopp, S., N’Guessan, N. A., Silué, K. D., Fürst, T., Lohourignon, L. K., Brou, J. K., N'Gbesso, Y. K., Vounatsou, P., N'Goran, E. K. and Utzinger, J. (2011). Accuracy of urine circulating cathodic antigen (CCA) test for Schistosoma mansoni diagnosis in different settings of Côte d'Ivoire. PLoS Neglected Tropical Diseases 5, e1384.

Coulibaly, J.T., Fürst, T., Silué, K. D., Knopp, S., Hauri, D., Ouattara, M., Utzinger, J. and N'Goran, E. K. (2012). Intestinal parasitic infections in schoolchildren in different settings of Côte d'Ivoire: effect of diagnostic approach and implications for control. Parasites \& Vectors $\mathbf{5}, 135$. Coulibaly, J. T., N'Gbesso, Y. K., N'Guessan, N. A., Winkler, M. S., Utzinger, J. and N'Goran, E. K. (2013). Epidemiology of schistosomiasis in two high-risk communities of south Côte d'Ivoire with particular emphasis on pre-school-aged children. American Gournal of Tropical Medicine and Hygiene 89, 32-41.

de Vlas, S. J. and Gryseels, B. (1992). Underestimation of Schistosoma mansoni prevalences. Parasitology Today 8, 274-277.

Greenbaum, A., Sikora, U. and Ozcan, A. (2012). Field-portable widefield microscopy of dense samples using multi-height pixel super-resolution based lensfree imaging. Lab on a Chip 12, 1242-1245.

Gryseels, B., Polman, K., Clerinx, J. and Kestens, L. (2006). Human schistosomiasis. Lancet 368, 1106-1118.

Gyorkos, T. W., Ramsan, M., Foum, A. and Khamis, I. S. (2001). Efficacy of new low-cost filtration device for recovering Schistosoma haematobium eggs from urine. Fournal of Clinical Microbiology 39, 26812682.

Hotez, P. J., Brindley, P. J., Bethony, J. M., King, C. H., Pearce, E. J. and Jacobson, J. (2008). Helminth infections: the great neglected tropical diseases. Fournal of Clinical Investigation 118, 1311-1321.

Isikman, S. O., Greenbaum, A., Lee, M., Bishara, W., Mudanyali, O., Su, T.-W. and Ozcan, A. (2012). Lensfree computational microscopy tools for cell and tissue imaging at the point-of-care and in low-resource settings. Analytical Cellular Pathology (Amsterdam) 35, 229-247.

Katz, N., Chaves, A. and Pellegrino, J. (1972). A simple device for quantitative stool thick-smear technique in schistosomiasis mansoni. Revista do Instituto de Medicina Tropical de São Paulo 14, 397-400.

Keiser, J., N'Goran, E. K., Traoré, M., Lohourignon, K. L., Singer, B. H., Lengeler, C., Tanner, M. and Utzinger, J. (2002). Polyparasitism with Schistosoma mansoni, geohelminths, and intestinal protozoa in rural Côte d'Ivoire. Fournal of Parasitology 88, 461-466.

Knopp, S., Mgeni, A. F., Khamis, I. S., Steinmann, P., Stothard, J. R., Rollinson, D., Marti, H. and Utzinger, J. (2008). Diagnosis of soiltransmitted helminths in the era of preventive chemotherapy: effect of multiple stool sampling and use of different diagnostic techniques. PLoS Neglected Tropical Diseases 2, e331.

Knopp, S., Becker, S. L., Ingram, K. J., Keiser, J. and Utzinger, J. (2013). Diagnosis and treatment of schistosomiasis in children in the era of intensified control. Expert Review of Anti-Infective Therapy 11, 1237-1258. Kongs, A., Marks, G., Verlé, P. and Van der Stuyft, P. (2001). The unreliability of the Kato-Katz technique limits its usefulness for evaluating S. mansoni infections. Tropical Medicine and International Health 6 , 163-169.

Kosinski, K. C., Bosompem, K. M., Stadecker, M. J., Wagner, A. D., Plummer, J., Durant, J. L. and Gute, D. M. (2011). Diagnostic accuracy of urine filtration and dipstick tests for Schistosoma haematobium infection in a lightly infected population of Ghanaian schoolchildren. Acta Tropica 118, 123-127.

Lee, M., Yaglidere, O. and Ozcan, A. (2011). Field-portable reflection and transmission microscopy based on lensless holography. Biomedical Optics Express 2, 2721-2730.

Linder, E., Grote, A., Varjo, S., Linder, N., Lebbad, M., Lundin, M., Diwan, V., Hannuksela, J. and Lundin, J. (2013). On-chip imaging of Schistosoma haematobium eggs in urine for diagnosis by computer vision. PLoS Neglected Tropical Diseases 7, e2547. 
Mott, K. E., Dixon, H., Osei-Tutu, E. and England, E. C. (1983). Relation between intensity of Schistosoma haematobium infection and clinical haematuria and proteinuria. Lancet 1, 1005-1008.

Murray, C. J. L., Vos, T., Lozano, R., Naghavi, M., Flaxman, A.D., Michaud, C. et al. (2012). Disability-adjusted life years (DALYs) for 291 diseases and injuries in 21 regions, 1990-2010: a systematic analysis for the Global Burden of Disease Study 2010. Lancet 380, 2197-2223.

Peters, P.A., Mahmoud, A.A., Warren, K.S., Ouma, J.H. and Siongok, T.K. (1976). Field studies of a rapid, accurate means of quantifying Schistosoma haematobium eggs in urine samples. Bulletin of the World Health Organization 54, 159-162.

Robinson, E., Picon, D., Sturrock, H. J., Sabasio, A., Lado, M., Kolaczinski, J. and Brooker, S. (2009). The performance of haematuria reagent strips for the rapid mapping of urinary schistosomiasis: field experience from Southern Sudan. Tropical Medicine and International Health 14, 1484-1487.

Ross, A. G. P., Bartley, P. B., Sleigh, A. C., Olds, G. R., Li, Y., Williams, G. M. and McManus, D. P. (2002). Schistosomiasis. New England Fournal of Medicine 346, 1212-1220.

Smith, Z. J., Chu, K., Espenson, A. R., Rahimzadeh, M., Gryshuk, A., Molinaro, M., Dwyre, D. M., Lane, S., Matthews, D. and Wachsmann-Hogiu, S. (2011). Cell-phone-based platform for biomedical device development and education applications. PLoS One 6, e17150.

Speich, B., Knopp, S., Mohammed, K. A., Khamis, I. S., Rinaldi, L., Cringoli, G., Rollinson, D. and Utzinger, J. (2010). Comparative cost assessment of the Kato-Katz and FLOTAC techniques for soil-transmitted helminth diagnosis in epidemiological surveys. Parasites $E^{\circ}$ Vectors 3, 71.

Steinmann, P., Keiser, J., Bos, R., Tanner, M. and Utzinger, J. (2006). Schistosomiasis and water resources development: systematic review, meta-analysis, and estimates of people at risk. Lancet Infectious Diseases 6, 411-425.

Steinmann, P., Utzinger, J., Du, Z.-W. and Zhou, X.-N. (2010). Multiparasitism: a neglected reality on global, regional and local scale. Advances in Parasitology 73, 21-50.

Stothard, J. R., Kabatereine, N. B., Tukahebwa, E. M., Kazibwe, F., Mathieson, W., Webster, J. P. and Fenwick, A. (2005). Field evaluation of the Meade Readiview handheld microscope for diagnosis of intestinal schistosomiasis in Ugandan school children. American fournal of Tropical Medicine and Hygiene 73, 949-955.

Tuijn, C. J., Hoefman, B. J., van Beijma, H., Oskam, L. and Chevrollier, N. (2011). Data and image transfer using mobile phones to strengthen microscopy-based diagnostic services in low and middle income country laboratories. PLoS One 6, e28348.

Utzinger, J., Booth, M., N'Goran, E. K., Müller, I., Tanner, M. and Lengeler, C. (2001). Relative contribution of day-to-day and intraspecimen variation in faecal egg counts of Schistosoma mansoni before and after treatment with praziquantel. Parasitology 122, 537-544.

WHO (2002). Prevention and control of schistosomiasis and soiltransmitted helminthiasis: a report of a WHO expert committee. $W H O$ Technical Report Series $\mathbf{9 1 2}, 1-57$. 